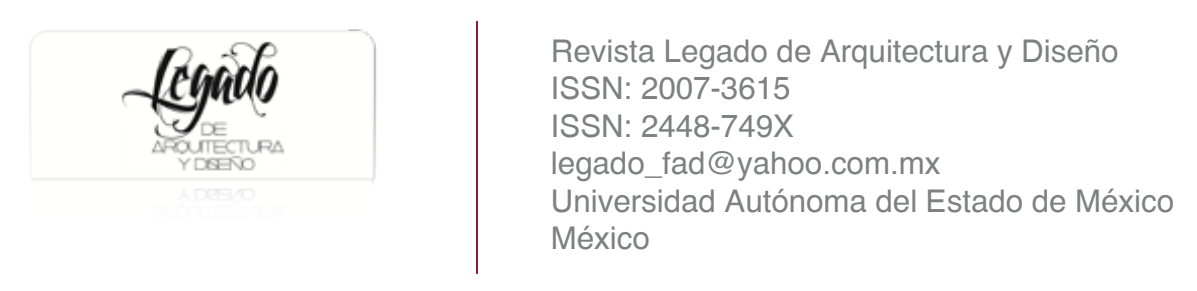

\title{
DISEÑO URBANO INTELIGENTE, HACIA UNA PROPUESTA DE ANÁLISIS E INTERVENCIÓN
}

Alva-Fuentes, Benjamín Fidel; Nava-López, Alfonso

DISEÑO URBANO INTELIGENTE, HACIA UNA PROPUESTA DE ANÁLISIS E INTERVENCIÓN

Revista Legado de Arquitectura y Diseño, vol. 2019, núm. 26, 2019

Universidad Autónoma del Estado de México, México

Disponible en: http://www.redalyc.org/articulo.oa?id=477961406011

Esta obra está bajo una Licencia Creative Commons Atribución-NoComercial-SinDerivar 4.0 Internacional. 


\title{
DISEÑO URBANO INTELIGENTE, HACIA UNA PROPUESTA DE ANÁLISIS E INTERVENCIÓN
}

\author{
SMART URBAN DESIGN, TOWARDS A PROPOSAL \\ FOR ANALYSIS AND INTERVENTION
}

\author{
Benjamín Fidel Alva-Fuentes benjamin.alva@uaslp.mx \\ Universidad Autónoma de San Luis Potosí, México \\ Alfonso Nava-López alfonsonava3@gmail.com \\ Universidad Autónoma de San Luis Potosí, México
} Revista Legado de Arquitectura y Diseño,
vol. 2019, núm. 26, 2019

Universidad Autónoma del Estado de México, México

Recepción: 02 Abril 2019

Aprobación: 23 Mayo 2019

Redalyc: http://www.redalyc.org/ articulo.oa?id $=477961406011$
Resumen: El avance de la tecnología ha cambiado radicalmente la vida de las personas y modificado la planeación, el diseño, la organización y la gestión de la ciudad. Los dispositivos móviles permiten la comunicación estrecha entre gobierno y sociedad, es posible la generación de datos en tiempo real para la toma de decisiones, la hiperconectividad, el Bigdata o el Internet de las cosas son conceptos que han surgido de la sofisticada generación, procesamiento, aprovechamiento de información y han calificado muchos sustantivos como inteligente: teléfonos inteligentes, televisiones inteligentes, automóviles inteligentes, casas inteligentes, e incluso ciudades inteligentes, pero ¿Cómo diseñar una ciudad inteligente?

Es fundamental desarrollar herramientas que adecuen la planeación y el diseño de las ciudades contemporáneas, por lo que este artículo busca abonar en la discusión de la ciudad inteligente y proponer un proceso de análisis para su diseño a partir de dos metodologías, una del Banco Interamericano de Desarrollo (BID) y otra de Diseño Urbano Centrado en las Personas; se ejempli fica el proceso de análisis con la Zona Industrial de San Luis Potosí, México. El análisis de caso muestra una metodología en construcción que ayudó a generar un proyecto de diseño urbano impulsado por el sector industrial. Este artículo muestra algunos resultados de un proyecto más amplio sobre movilidad inteligente que se realiza en conjunto entre la Universidad Autónoma de San Luis Potosí y la Unión de Usuarios de la Zona Industrial.

Palabras clave: Diseño urbano, ciudad inteligente, metodología de diseño, uso de datos. Abstract: The advancement of technology has changed the way of people's lives and modified planning, design, organization and city management. Mobile devices allow close communication between government and society, it's possible generate real-time data for decision making, hyperconnectivity, Bigdata or the Internet of things are concepts that have emerged from the sophisticated generation, processing, leveraging information and have qualified many nouns such as smart like smart phones, smart $T V$ s, smart cars, smart homes, and even smart cities, but how to design a smart city?

It's essential to develop tools that allow planning and design of contemporary cities, this document seeks to proceed in the discussion of the smart city and propose an analysis process for its design since two methodologies, one of the Inter-American Development Bank (IDB) and another of People-centered Urban Design; It exemplifies the study process with the Industrial Zone of San Luis Potosi, Mexico. The analysis in the case of study shows a methodology under construction that helped to generate an urban design project driven by the industrial sector. This article shows part of results of the research project about smart mobility that is carried out jointly between the Autonomous University of San Luis Potosi and the Users Union of the Industrial Zone.

Keywords: Urban design, smart city, methodology design, data application in design . 


\section{INTRODUCCIÓN}

Diversas iniciativas del Banco Interamericano de Desarrollo (BID), de gobiernos nacionales y de organizaciones civiles buscan transformar con éxito las ciudades tradicionales para ser inteligentes, es decir, espacios urbanos capaces de generar datos, utilizarlos para la toma de decisiones y solucionar problemas, es decir, ser más efectivos o sustentables.

El objetivo de este artículo es integrar el concepto de "inteligente" al diseño urbano y esquematizar un proceso de análisis para intervenir en la ciudad. La propuesta se integra con dos metodologías, la estrategia del BID para las ciudades inteligentes y una idea en construcción sobre la metodología de Diseño Urbano Orientado a las Personas. ${ }^{[1]}$ En la primera parte se abona la discusión conceptual de la ciudad inteligente; en la segunda se describe brevemente la metodología, en la tercera se presenta un caso de estudio y a manera de conclusión se describen algunos retos en la implementación de este proceso de análisis que podría representar una metodología en construcción.

\section{La ciudad inteligente}

A partir de este siglo se han multiplicado las iniciativas para la mejor planeación o diseño de la ciudad mediante el uso del conocimiento o las Tecnologías de la Información y la Comunicación (TIC), por ejemplo, la ciudad de la información, la ciudad cibernética, la ciudad conectada, la ciudad digital, la ciudad del conocimiento, la ciudad innovadora y ahora la ciudad inteligente (Kitchin, 2014).

La diversidad de adjetivos para la ciudad ha llevado a la literatura especializada a desarrollar el entendimiento de cada uno; después, la forma cómo se mide, mientras que la construcción de un método que permita generar esos modelos de ciudad se ha mantenido un tanto rezagada. La ciudad inteligente tiene sus referentes en los estudios de Hall (2000), Chourabi et al. (2012), Kitchin (2014), Chiehyeon (2018); el desarrollo de formas de medición e índices para medir la ciudad inteligente ha estado presente en los estudios de Giffinger (2007), Chen, Chiang y Storey (2012); mientras que el método tiene un estudio más completo en la Iniciativa de Ciudades Emergentes y Sostenibles del BID (2016).

\section{La generación, gestión y aprovechamiento de datos QUE SE CONCENTRAN EN LA CIUDAD REDIMENSIONA EL ANÁLISIS URBANO PARA INTEGRAR EL ESPACIO DIGITAL CON EL FÍSICOEN MÚLTIPLES FORMAS.}

A pesar de los diversos avances en el desarrollo del concepto, es necesario realizar algunas precisiones con el propósito de abonar en la discusión y profundizar en la construcción de métodos y procesos de análisis y diseño. En este apartado se realiza una breve descripción del 
aprovechamiento de datos en la gestión de la ciudad, para posteriormente acotar el concepto de ciudad inteligente.

El uso intensivo de la tecnología y los datos tiene diferentes escalas espaciales, desde lo virtual hasta lo físico, desde la vivienda hasta el país, pasando por el barrio, el sector, el distrito, la ciudad, la metrópoli o la región; ambos son esenciales para la comunicación entre personas (Achaerandio, 2011). La ciudad también genera un conjunto infinito de datos y combinaciones que pueden ser recolectados y analizados en tiempo real, con el fin de tomar mejores decisiones de una manera más rápida (Kitchin, 2013). La correcta utilización de datos masivos apoya el mejor entendimiento de fenómenos urbanos, la generación del conocimiento y la toma de decisiones (Sevillano, 2015).

Una gestión urbana basada en datos generados "en tiempo real" permite mejorar la efectividad de los procesos, servicios y la gobernanza (2012); un gobierno inteligente, por ejemplo, incluye sistemas integrados de administración y comunicación para la solución de problemas (Bélissent, 2010).

Así, la generación, gestión y aprovechamiento de datos que se concentran en la ciudad redimensiona el análisis urbano para integrar el espacio digital con el físico en múltiples formas. El uso intensivo de las TIC la generación y utilización de datos masivos que intercambian las personas y su aprovechamiento en conocimiento hacen posible que una ciudad sea inteligente.

El término de ciudad inteligente tiene sus antecedentes en la literatura de la década de los ochenta del siglo XX. Desde su origen, el desarrollo del concepto ha variado entre países y organizaciones, en este siglo se han desarrollado significados y usos más específicos (Nicos \& Mora, 2018), tal es el caso de la solución de problemas de sostenibilidad centrados en la eficiencia energética, reducción de emisiones de carbono (Achaerandio, 2011) o mejorar la vida urbana a través de sistemas inteligentes de transporte o de seguridad (Giffinger, 2007).

En 1994 más de 100 organizaciones europeas comenzaron a discutir el término de ciudad inteligente asociado con aplicaciones telemáticas como la movilidad (Ishida, 2000). A partir de este siglo, el término se vinculó a la disponibilidad y capacidad de infraestructura de telecomunicaciones en la ciudad (Achaerandio, 2011).

El BID (2016) define a la ciudad inteligente como el espacio urbano que coloca a las personas en el centro del desarrollo, incorpora TIC en la gestión urbana e incluye procesos de planificación colaborativa y participación ciudadana. Autores como Lipman (1986), Hall (2000) y Chourabi (2012) coinciden en que la ciudad inteligente es un conjunto de infraestructura y elementos tecnológicos capaces de establecer redes que conecten de forma física y virtual a las personas. Particularmente, Hall (2000) señala que, la ciudad inteligente es una red de estructuras conectadas entre sí a partir de sistemas avanzados e integrados, que recolectan datos en tiempo real con el fin de mejorar la gestión de la ciudad. 
En este sentido, la ciudad inteligente requiere procesos que faciliten la transformación urbana a partir de la colaboración de agentes a diversas escalas (Komninos, Tsarchoupoulos, Kakderi, 2014). En esencia, es gestión de datos, del espacio y comunicación entre personas (Achaerandio, 2011).

En la ciudad inteligente la infraestructura genera y comparte información con otros subsistemas a través de telecomunicaciones, sensores que recolectan información y sistemas robustos de procesamiento de datos que ordenan, sintetizan, e interpretan información; representa la capacidad cognitiva del sistema urbano (Chourabi et al., 2012).

Una ciudad inteligente se entiende desde la perspectiva social por la interacción entre personas que forman parte activa en las decisiones, la sociedad genera información y conocimiento que les permite actuar de manera colectiva ante cualquier cambio (Ishida, 2000); es una forma de organización urbana para percibir cambios en el entorno y responder de forma efectiva (Liugailaite \& Jucevicius, 2014).

La ciudad inteligente está compuesta por elementos tecnológicos y sociales que, integrados, funcionan como herramienta para la gestión de espacios urbanos (Giffinger, 2007). Los principales elementos son: la red, datos masivos, conocimiento, innovación, actividades y relaciones.

A partir de las definiciones anteriores y a manera de síntesis, una ciudad inteligente genera datos en tiempo real y conocimiento a partir de las actividades y uso de infraestructura, establece entornos conectados y accesibles, y coloca a las personas en el centro del desarrollo urbano, para involucrarlos en la toma de decisiones; inicialmente requiere un propósito, preferentemente la sustentabilidad (Kitchin, 2014).

Es importante precisar que si bien las definiciones de una ciudad inteligente son variadas, aún se considera una categoría emergente con diferentes nombres y circunstancias (Nam \& Pardo, 2011). Así, aunque hace falta precisión y no existe consenso sobre la definición de una ciudad inteligente, se consideran términos de convergencia la infraestructura, el uso de la tecnología, la gestión del conocimiento , y cómo se relacionan las personas. La infraestructura es la base sobre la cual se establece la red de relaciones físicas y virtuales, la tecnología se emplea para generar y administrar datos, y el conocimiento es un valor agregado en la solución de problemas.

\section{La infraestructura es la base sobre la cual se establece \\ LA RED DE RELACIONES FÍSICAS Y VIRTUALES, LA TECNOLOGIA SE EMPLEA PARA GENERAR Y ADMINISTRAR DATOS, $y$ el conocimiento es un valor agregado en la solución de problemas.}

\section{Hacia un Diseño Urbano Inteligente (DUI)}

A partir de las premisas sobre el uso de datos, la generación de redes y relaciones, la centralidad en las personas y otros términos asociados al concepto de ciudad inteligente, así como de la fusión de dos metodologías 
una de Diseño Urbano Centrado en la Persona ${ }^{[2]}$ y otra del BID, ${ }^{[3]}$ se estructura un proceso de análisis para el diseño urbano basado en datos ordenados en seis redes, cada una representa una plataforma ordenada secuencialmente. La red parte de una retícula de tres niveles (figura 1), una base agrupa los elementos que dan soporte a los proyectos de ciudad, el segundo se enfoca a la persona colocandola en el centro del diseño y el tercero a los fines que busca la ciudad.

Partiendo de la premisa que la inteligencia está basada en datos y que existen disciplinas que acunaron primero el término como la tecnología, se aplica también el concepto de Procesamiento Analítico en Línea (OLAP, por sus siglas en inglés); bajo este modelo es posible almacenar información y analizarla desde múltiples plataformas; así el cubo de datos resultó ser la figura más adecuada (figura 1).
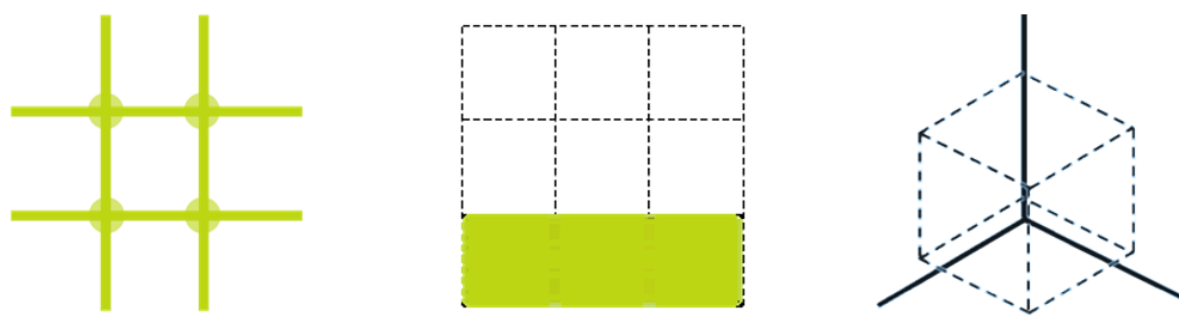

Figura 1. Conceptualización del Diseño Urbano Inteligente (DUI).

Fuente: Elaboración propia.

De las seis plataformas consideradas, cuatro son de diagnóstico y dos de propuesta. Un cubo permite asociar las seis plataformas, su construcción es un proceso de ensamble tridimensional que permite observar el comportamiento de cada lado, su conjunto y las posibilidades de intercambio y dinámica al análisis. Además, con la analogía del Cubo Rubik $^{[4]}$ se asigna códigos de color a cada plataforma (figura 2) a partir de:

1. Redes: La fila inferior es la información en tiempo real, la tecnología y telecomunicaciones para generar conocimiento. En la segunda fila, se encuentra el equipamiento, los procesos y la seguridad adecuada para que las personas realicen actividades. La tercera como fin, muestra elementos de enlace, nodos ubicados en tiempo y lugar que concentran actividades y relaciones de personas, interacciones en los espacios urbanos y morfología de la ciudad.

2. Actividades: En la fila base se encuentran los servicios gubernamentales, financieros y educativos; en la segunda fila los servicios recreativos, espacio público y cultural; en la parte superior la industria, el comercio o la actividad motriz de la ciudad, es decir, representan las actividades que generan mayor empleo.

3. Alianzas y liderazgo: En la base se ubica el diagnóstico de las capacidades, se refiere a los servicios digitales, la vocación para la innovación (disponibilidad de centros o 
clústeres con capacidad de desarrollar tecnología, investigación y conocimiento), así como los proveedores de tecnología. En la fila de las personas, se identifica el nivel de involucramiento social en la toma de decisiones, y, en la fila propósito se reconocen los líderes del proyecto, un equipo interdisciplinario que posibilita realizar alguna intervención y la posibilidad de gestión.

4. Financiamiento: En la base se ubican las fuentes de recursos disponibles y potenciales. En el centro, los costos que asumen las personas de forma individual, colectiva o los proyectos detonantes de la economía. El propósito está representado por los beneficios en términos económicos, sociales y territoriales.

Con estas cuatro dimensiones, se establece un diagnóstico con datos que incluyen tres niveles, la base para el desarrollo de la ciudad, las personas como beneficiarios y las relaciones, así como los fines o propósitos que busca cada dimensión. Las dos siguientes plataformas están asociadas al diseño, primero del espacio y posteriormente de la marca que permita el convencimiento del proyecto.

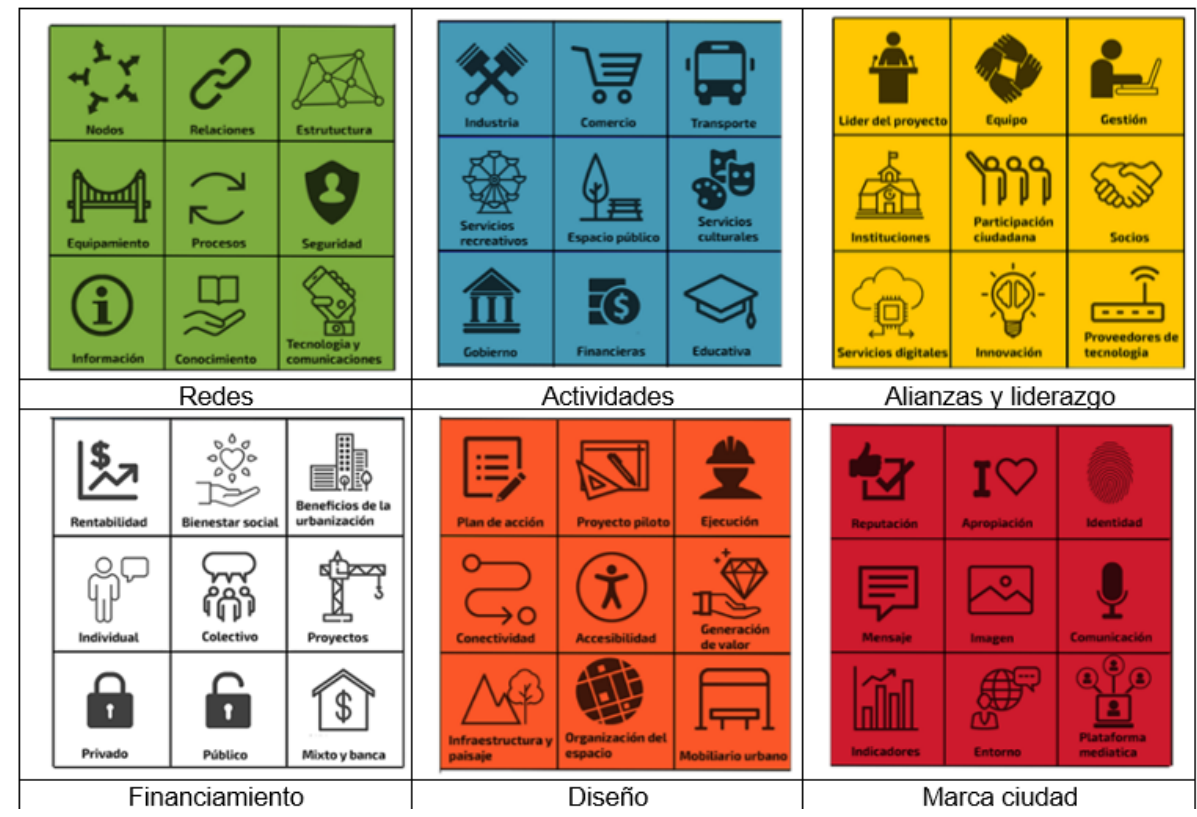

Figura 2. Plataformas del Diseño Urbano Inteligente (DUI).

Fuente: Elaboración propia a partir de metodología de ciudades inteligentes del Banco Interamericano de Desarrollo (2016).

1. Diseño: Esta plataforma representa espacios y criterios ordenados, en la base la infraestructura, el paisaje, el ordenamiento del territorio y del espacio virtual; en el centro las personas mediante criterios de conectividad, accesibilidad y generación de valor que debe provocar el proyecto, en la fila de resultados se presenta el plan de acción hasta la ejecución, constituye la estrategia rectora o ruta de corto, mediano y largo plazos.

2. Marca ciudad: En la primera fila se construyen los indicadores, entorno mediático y plataformas; en la fila central se especifica 
la comunicación, integrada por el mensaje, la imagen y el slogan; finalmente como resultado se plantea la consolidación de una marca representada por la percepción positiva de la imagen, confianza, apropiación y arraigo sociocultural, en términos generales el Branding del diseño urbano.

De esta manera, el ensamble de las plataformas permite un análisis de izquierda a derecha y de abajo hacia arriba (figura 3). En el diseño inteligente, las personas en el centro, obliga a pensar en relaciones, actividades, procesos basadas en datos.

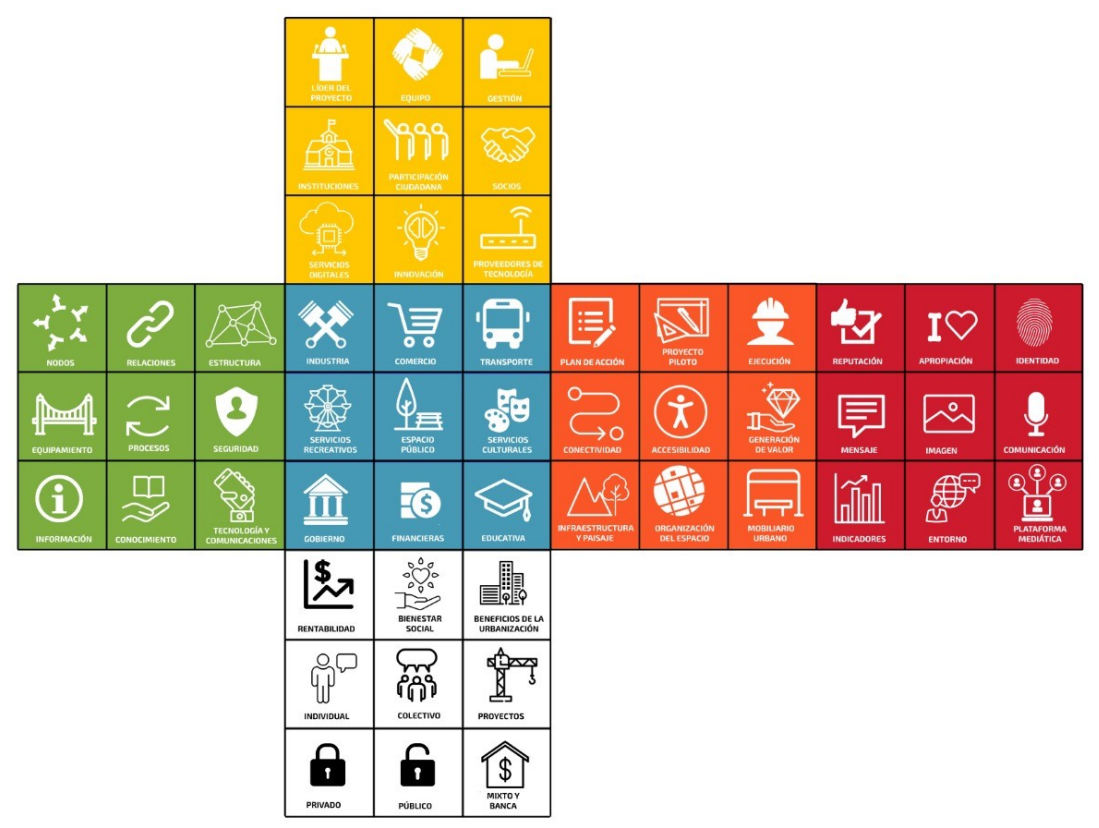

Figura 3. Integración de plataformas para el Diseño Urbano Inteligente (DUI).

Fuente: Elaboración propia a partir de metodología de ciudades inteligentes del Banco Interamericano de Desarrollo (2016).

Con este orden, los datos se transforman en estrategias de conectividad, accesibilidad y generación de valor, por tanto, las plataformas, la red y cada cuadro comienza a girar para agrupar los valores del diagnóstico ubicados en diversas plataformas, en un lado único (figura 4). Al analizar de manera transversal las plataformas, el movimiento del cubo de datos asocia elementos con bajo valor, o con importancia estratégica, coloca en el centro el elemento de diseño que permita explicar la lógica de la agrupación en sentido dextrógiro. ${ }^{[5]}$ 

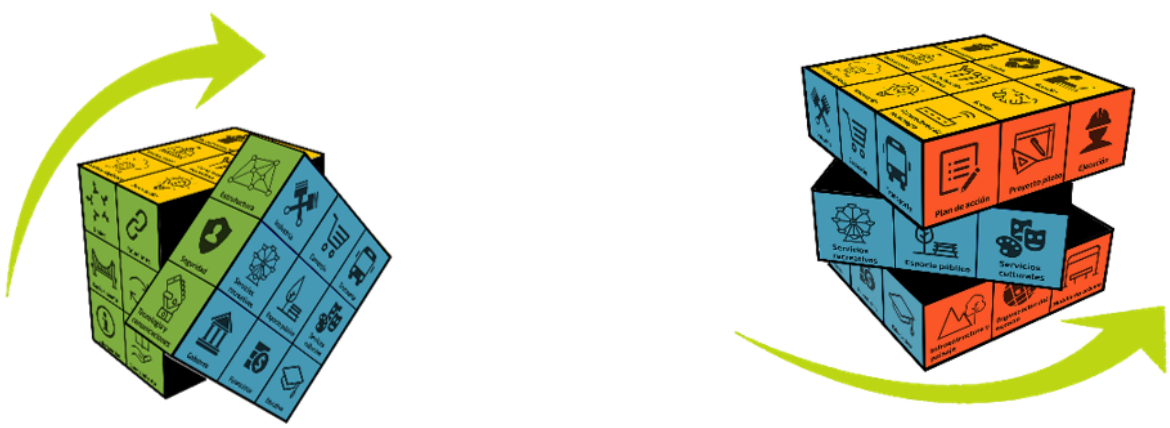

Figura 4. De la estrategia a los datos, proceso de DUI.

Fuente: Elaboración propia.

A esta forma de agrupación con diversos elementos de las plataformas ordenados a partir de un elemento de diseño se denomina "dimensión". ${ }^{[6]}$ Así, es posible asociar y ordenar un grupo de datos bajo un criterio estratégico, por ejemplo, mejorar valores, en un ejercicio hipotético los datos obtenidos en la fase de diagnóstico relacionados con conectividad son (figura 5):

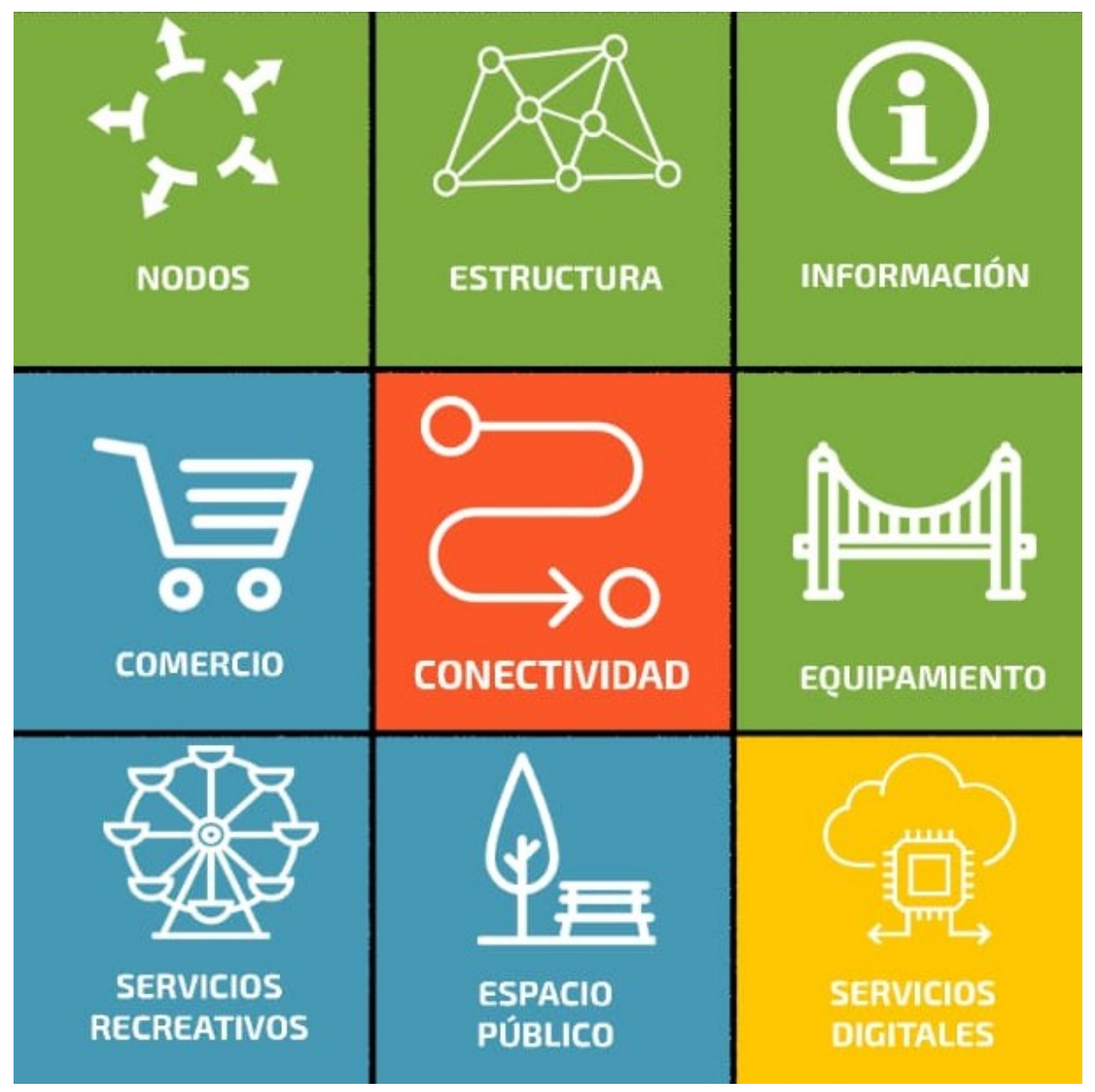

Figura 5. De la estrategia a los datos, proceso de DUI.

Fuente: Elaboración propia.

Finalmente, con las dimensiones definidas se construye la plataforma de la marca ciudad. Es importante precisar que la dimensión puede cambiar durante el proceso de diseño o de la marca, como consecuencia 
de una variación en los datos o de la organización; de tal manera que el proceso es interactivo, así se puede observar cómo se modifican los indicadores y se construye la identidad y el mismo diseño se puede ajustar. El uso de los datos a lo largo del proceso, su interacción y la necesidad de tomar decisiones son las principales características que conforman el DUI.

A continuación se presenta la aplicación de la propuesta en un estudio realizado en la Zona Industrial de San Luis Potosí. Primero se realizó una robusta base de datos georreferenciada, alimentada con información del Instituto Nacional de Estadísticas y Geografía (INEGI), encuestas y entrevistas. Se analizó en un sistema informático y se obtuvieron los resultados de la figura 6 .

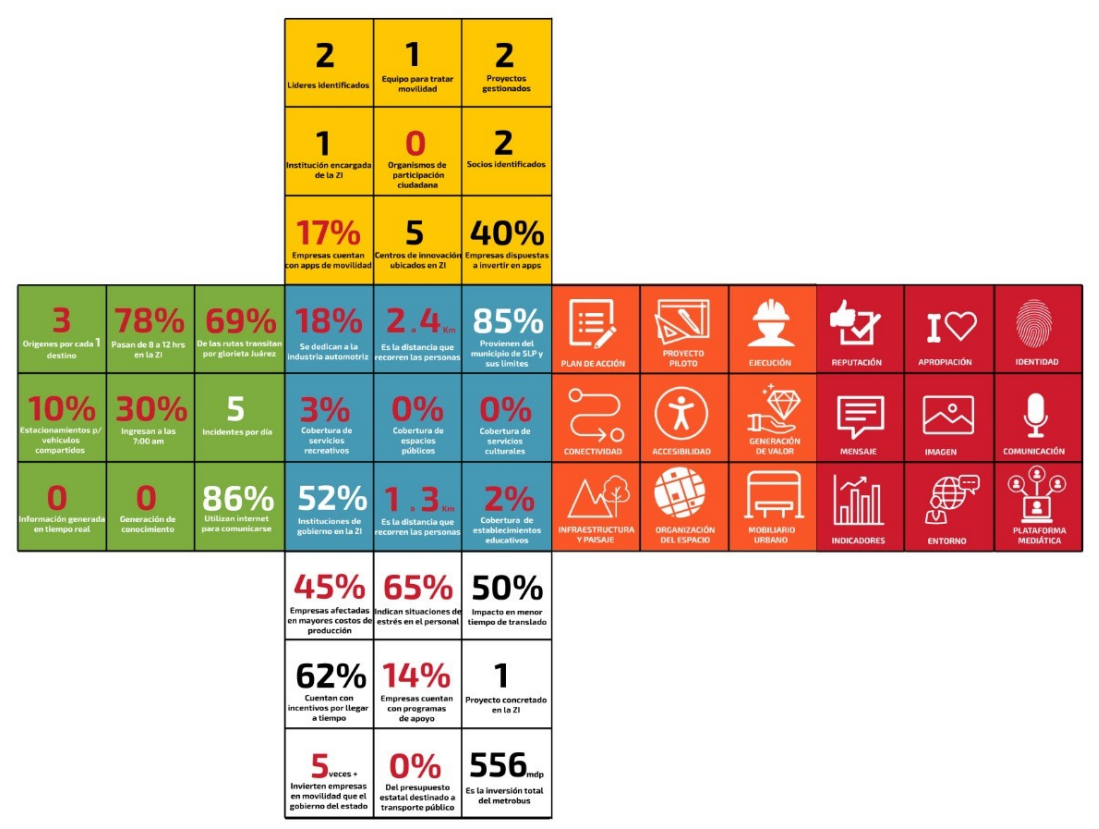

Figura 6. Datos de la Zona Industrial de San Luis Potosí, México.

Fuente: Elaboración propia con datos obtenidos del proyecto Movilidad Inteligente en la Zona Industrial.

Con relación a los nodos que existen en la ciudad, se identificaron 81 a partir del origen y destino de transporte de personal ubicados en la Zona Metropolitana de San Luis Potosí, 90\% se encuentran en el municipio de San Luis Potosí y se conectan por las vialidades de Avenida Salvador Nava, Carretera Matehuala, Carretera 57, Avenida Fray Diego y Avenida Salk, Avenida Industrias y los ejes 104, 114, 128, CFE, Ampliación y 140. Sin embargo, existe una relación de tres orígenes por cada un destino en la Zona Industrial, esto ocasiona un efecto embudo aumentando la movilidad y limitando la accesibilidad a la zona industrial.

A partir del análisis de las relaciones, destaca el hecho que las personas pasan de 8 a 12 horas en la Zona Industrial, contando el tiempo de traslado desde sus hogares al trabajo. En cuanto a las estructuras, $69 \%$ de las rutas de transporte público y de personal transitan por un solo punto, el distribuidor vial Benito Juárez. 
El equipamiento considera todo lo disponible para la realización de las actividades incluyendo la movilidad por tipo, destaca que solo $10 \%$ de las empresas cuentan con estacionamientos exclusivos para los trabajadores que comparten el auto; sin embargo, $38 \%$ de las personas que viajan en auto, estarían dispuestos a utilizar un servicio de bicicletas compartidas como alternativa de movilidad.

El análisis de los principales procesos permitió analizar las actividades cotidianas de las personas, $37 \%$ cuentan con tres turnos, $26 \%$ con solo un turno y $21 \%$ con dos turnos. Diariamente el $30 \%$ del personal operativo ingresa a su trabajo a las 7:00 am y el 20\% termina sus actividades laborales a las 3:00 pm, mientras que $58 \%$ del personal administrativo ingresa a las 8:00 am y el $49 \%$ termina a las 6:00 pm.

En materia de seguridad, ocurren 5 incidentes viales por día, los principales delitos están relacionados con el robo de autopartes, principalmente entre dos de las principales vialidades. Uno de los principales aspectos que disminuye la percepción de seguridad en la zona industrial es la falta de alumbrado público, únicamente $50 \%$ de las vialidades de la zona industrial disponen de luminarias.

El uso de las TIC es imprescindible en el diseño de una ciudad inteligente. Se identificó que 55\% de las personas encuestadas tenían acceso a Internet a través de sus áreas de trabajo, 38\% lo hacían mediante su teléfono celular y solo $7 \%$ desde su vivienda. El uso que le dan es determinante en la movilidad, $67 \%$ lo utilizaba para obtener información, no estaba disponible una aplicación para dispositivos moviles que hiciera más eficiente la movilidad en la zona industrial, solo 2 empresas que habían desarrollado la propia.

Con cifras del DENUE del INEGI se mapearon 327 empresas manufactureras , 46 comercios distribuidos en tres secciones , 50\% de ellos concentrados en la primera parte. La distancia que recorren las personas entre comercios es de $350 \mathrm{~m}$ en promedio, los límites de una distancia caminable es de $400 \mathrm{~m}$; sin embargo, existen áreas en la zona industrial que resultan inaccesibles para las personas que trabajan en dos terceras partes de la distribución. 


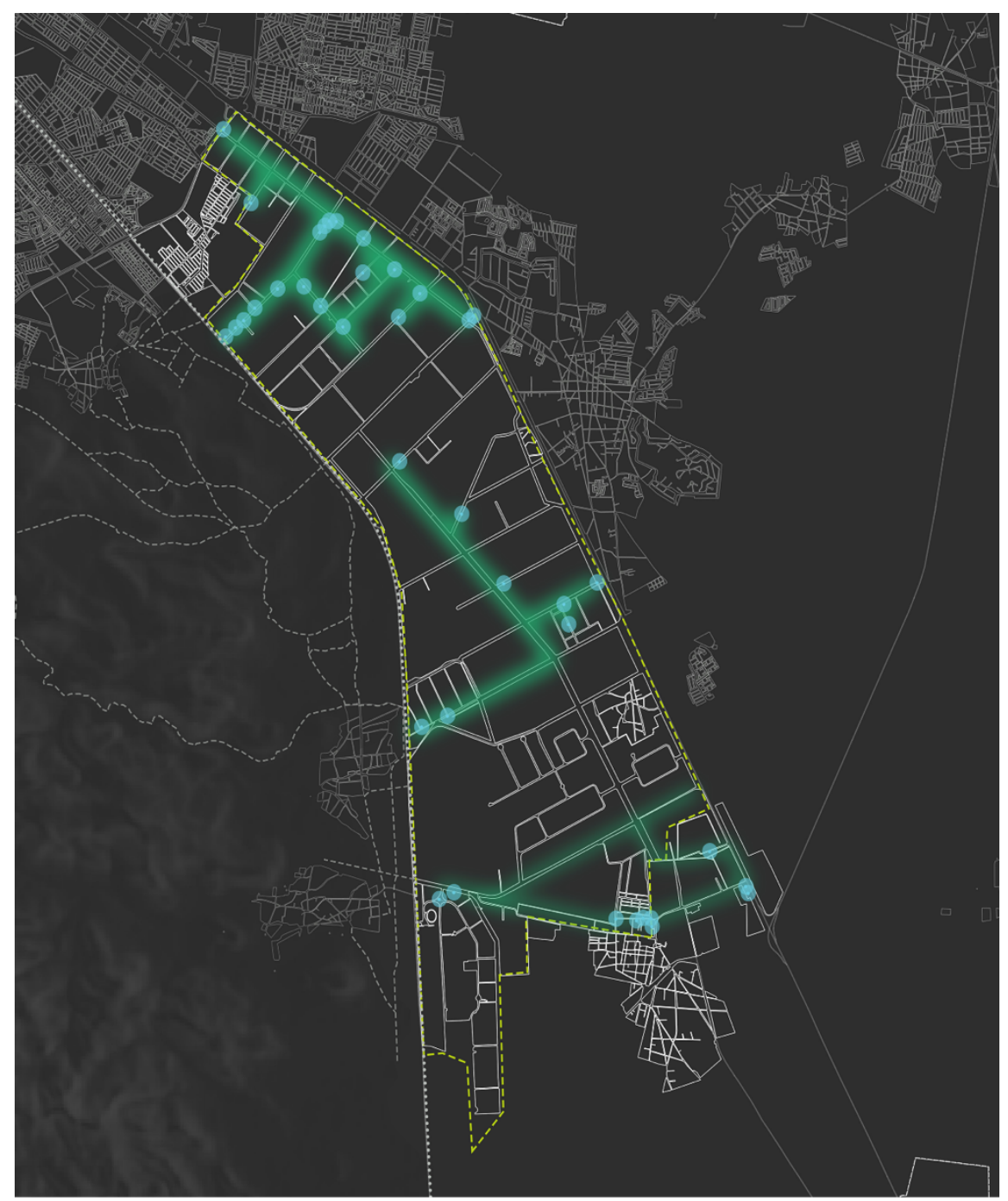

Figura 7. Distribución de los centos comerciales y desplazamientos de las personas en la Zona Industrial de San Luis Potosí.

Fuente: Elaboración propia.

A manera de ejemplo se presentan las cifras más representativas de los indicadores que se observaron en el diagnóstico, empleando el Diseño Urbano Inteligente, posteriormente se hicieron combinaciones destacando cuatro dimensiones: accesibilidad, espacio público, infraestructura del paisaje y generación de valor.
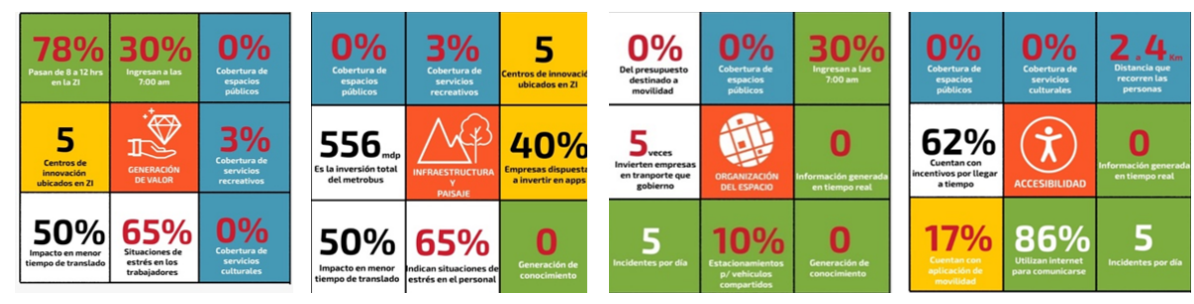

Figura 8. Dimensiones del Diseño urbano Inteligente para la Zona Industrial. Fuente: Elaboración propia.

Como última parte, se realizó una marca ciudad y algunas propuestas de diseño partiendo de los criterios de cada dimensión analizada y con 
la finalidad de mejorar el valor de los indicadores, las relaciones y las actividades de las personas.
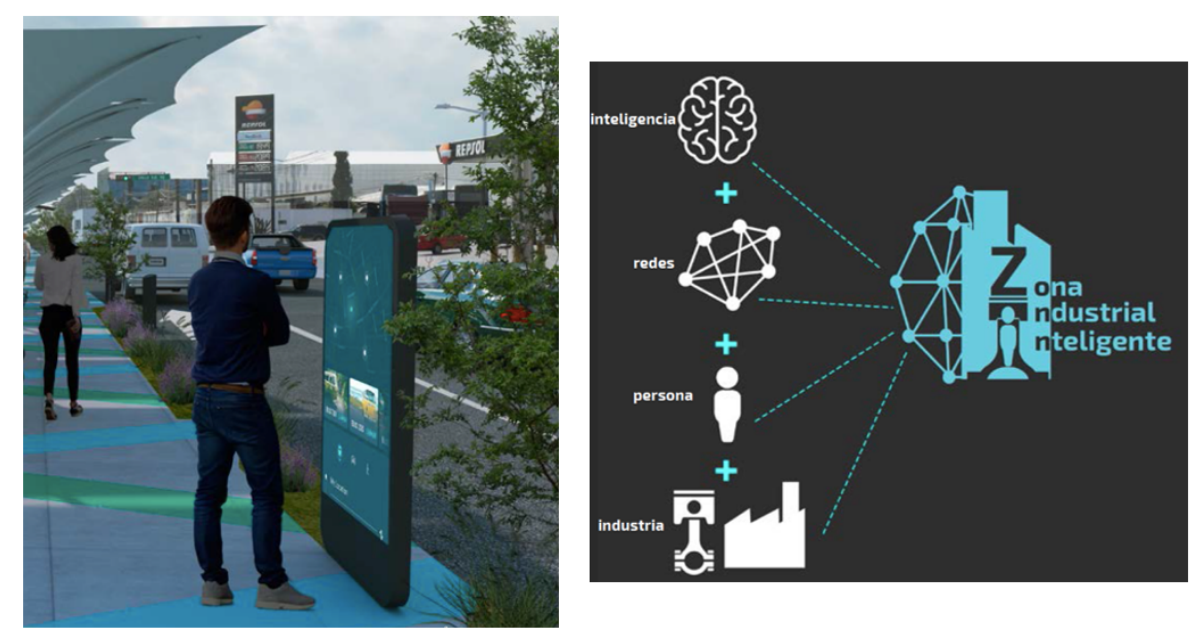

Figura 9. Ejemplo de Diseño Urbano Inteligente y Marca Ciudad.

Fuente: Elaboración propia.

Así, es factible introducir el manejo de datos dinámico en el análisis del entorno a partir de 4 plataformas de diagnóstico y generar multiples combinaciones de dimensiones con 9 criterios establecidos por esta propuesta de análisis para la generación de ciudades inteligentes. En el caso de la Zona Industrial de la Ciudad de San Luis Potosí, México, permitió hacer un análisis con variables novedosas, procesar una amplia cantidad de información y sintetizarla en criterios útiles para el tipo de ciudad propuesto, pero sobre todo, organizar mejor el espacio urbano, agregar valor y establecer una marca que permita posicionar mejor al proyecto para el concurso de recursos, la participación de los diversos agentes y de la ciudadanía.

\section{CONCLUSIONES}

El Diseño Urbano Inteligente (DUI) es una propuesta que busca cambiar la gestión del diseño a partir del uso de datos y el empleo de tecnologías. Si planteamos construir ciudades inteligentes ¿cómo las diseñamos?, justamente de esa pregunta surge esta propuesta que pretende abonar en la construcción de conceptos y métodos que modifiquen la forma como planeamos y diseñamos las ciudades. Esta propuesta de DUI proporciona una herramienta que fusiona categorías emergentes como la centralidad en las personas, el uso de la tecnología, el conocimiento y la inteligencia.

Tal vez uno de los principales retos en la inteligencia aplicada en la ciudad y el diseño, es la calidad de la infraestructura, así como la generación y aprovechamiento de datos. La falta de infraestructura basada en tecnologías en las ciudades hace imposible la generación de datos, algunas ciudades inician con el gobierno digital, otras con el gobierno abierto, pero hace falta su vinculación con la toma de decisiones o con el diseño de obras para intervenir el espacio público. De esta manera, la disponibilidad de tecnología para generar, procesar y aprovechar los datos 
específicos sobre las relaciones en el espacio público representa la principal limitante del DUI.

La propuesta de DUI surgió justamente de una investigación que analizó la factibilidad de una ciudad inteligente en San Luis Potosí, México, como parte de la agenda se identificó la necesidad de contar con una metodología o al menos un nuevo proceso de análisis de información para el diseño urbano.

Si bien se reconocen las limitaciones del DUI y el carácter de construcción en la que se encuentra la metodología, también es importante afirmar que la tecnología permite disminuir las barreras, por ello, seguramente la próxima década existirán nuevas condiciones que permitan diseñar ciudades inteligentes.

Tal vez uno de los principales retos en

\section{LA INTELIGENCIA APLICADA EN LA CIUDAD Y EL DISEÑO, \\ ES LA CALIDAD DE LA INFRAESTRUCTURA, \\ así como la generación y aprovechamiento de datos.}

\section{FUENTES DE CONSULTA}

Achaerandio, R. (2011), Análisis de las ciudades inteligentes en España. [En línea] www.aeiciberseguridad.es: https://www.aeiciberseguridad.es/ descargas/categoria6/8883484.pdf, consultado el 5 de mayo de 2019.

Alva, B., Narvaez, F. \& Martinez, A. (2017), Diseño urbano centrado en la persona. Una propuesta para enfrentar los retos urbanos en América Latina. Planeo, 1-15. [En línea] http://revistaplaneo.cl/wp-content/ uploads/Arti\%CC\%81culo_AlvaNarvaezMartinez.pdf, consultado el 20 de mayo de 2019.

Banco Interamericano de Desarrollo (BID) (2016), La ruta hacia las Smart Cities. Banco Interamericano de Desarrollo. [En línea] https://publications.iadb.org/bitstream/handle/11319/7743/Laruta-hacia-las-smart-cities-Migrando-de-una-gestion-tradicional-a-laciudad-inteligente.pdf, consultado el 2 de abril de 2019.

Bélissent, J. (2010), Getting clever about smart cities. Retrieved from Forrester: http://193.40.244.77/iot/wp-content/uploads/2014/02/ getting_clever_about_smart_cities_new_opportunities.pdf, consultado el 14 de abril de 2019.

Chen, H., Chiang, R. H. \& Storey, V. C. (2012), Bussines intelligence and analytics: from big data to big impact. MIS Quarterly: Bussines intelligence research, pp. 1165-1188. Retrieved from https://pdfs.semanticscholar.org/f5fe/ b79e04b2e7b61d17a6df79a44faf358e60cd.pdf, consultado el 2 de junio de 2019.

Chiehyeon, L., Kim, K.-J. \& Maglio, P. (2018), Smart cities with big data: Reference models, challenges, and considerations, Cities, pp. 86-99.

Chourabi, H., Nam, T., Walker, S., Gil-Garcia, R., Mellouli, S., Nahon, K., Scholl, H. (2012), Understanding Smart Cities: An Integrative Framework, consultado el 18 de abril de 2019. 
Giffinger, R. (2007), Smart cities Ranking of European medium-sized cities. Retrieved from www.smart-cities.eu: http://www.smart-cities.eu/ download/smart_cities_final_report.pdf, consultado el 23 de mayo de 2019.

Hall, R. (2000), The Vision of A Smart City. Retrieved from osti.gov. https:// www.osti.gov/servlets/purl/773961, consultado el 2 de abril de 2019.

Ishida, T. (2000), Understanding Digital Cities. Retrieved from Department of Social Informatics, Kyoto University: http://citeseerx.ist.psu.edu/ viewdoc/download?doi=10.1.1.75.2175\&rep=rep1\&type=pdf, consultado el 14 de mayo de 2019.

Kitchin, R. (2013), The Real-Time City? Big Data and Smart Urbanism. Retrieved from SSRN Electronic Journal: https://www.researchgate.net/ publication/314410892_The_Real-

Time_City_Big_Data_and_Smart_Urbanism, consultado el 14 de abril de 2019.

Kitchin, R. (2014), Getting smarter about smart cities: Improving data privacy and data security. Retrieved from www.taoiseach.gov.ie: https://www.taoiseach.gov.ie/eng/Publications/ Publications_2016/Smart_Cities_Report_January_2016.pdf, consultado el 20 de mayo de 2019.

Komninos, N., Tsarchoupoulos , P. \& Kakderi, C. (2014), New Services Design for Smart Cities: A Planning Roadmap for User-Driven Innovation. Retrieved from URENIO Research: http://www.urenio. org/komninos/wp-content/ uploads/2014/08/New-services-design-forsmart-cities.pdf, consultado el 18 de mayo de 2019.

Lipman, A., Sugarman, A. \& Cushman, R. (1986), Teleports and the inteligent city. Retrieved from sugarlaw.com: http://www.sugarlaw.com/ publications/teleport/teleports-all-dow-jones.pdf, consultado el 2 de mayo de 2019.

Liugailaite, L. \& Jucevicius, R. (2014), Going to be an intelligent city. Retrieved from Elsevier: https://ac.els-cdn.com/S1877042814059515/1-s2.0S1877042814059515-main.pdf?_tid=163b8590-130f-11e8-

bb7b-00000aab0f26\&acdnat $=1518781796 \_$e2bcfe5ab6d48bd33561a0 054ba1bf7d, consultado el 3 de mayo de 2019.

Kitchin, R. (2013), The Real-Time City? Big Data and Smart Urbanism. Retrieved from SSRN Electronic

Journal:

publication/314410892_The_Real-

https://www.researchgate.net/

Time_City_Big_Data_and_Smart_Urbanism, consultado el 14 de abril de 2019.

Kitchin, R. (2014), Getting smarter about smart cities: Improving data privacy and data security. Retrieved from www.taoiseach.gov.ie: https://www.taoiseach.gov.ie/eng/Publications/ Publications_2016/Smart_Cities_Report_January_2016.pdf, consultado el 20 de mayo de 2019.

Komninos, N., Tsarchoupoulos, P. \& Kakderi, C. (2014), New Services Design for Smart Cities: A Planning Roadmap for User-Driven Innovation. Retrieved from 
URENIO Research: http://www.urenio.org/komninos/wp-content/ uploads/2014/08/New-services-design-for-smart-cities.pdf, consultado el 18 de mayo de 2019.

Nam, T. \& Pardo, T. (2011), Conceptualizing Smart City with Dimensions of Technology, People, and Institution, consultado el 14 de abril de 2019.

Nicos, K. \& Mora, L. (2018), Exploring the big picture of Smart City. Retrieved from ResearchGate: https://www.researchgate.net/ publication/319598847, consultado el 6 de mayo de 2019.

Sevillano, F. (2015), "Big data", Economia industrial, pp. 71-86.

\section{Notas}

[1] Para más detalle de esta propuesta véase Alva, Narváez y Martínez (2017).

[2] Para más información de esta propuesta ver Alva (2017).

[3] El detalle de la metodología se describe por el BID en el documento La Ruta hacia las Smart Cities (2016).

[4] Rompecabezas mecánico tridimensional inventado por el escultor y profesor de arquitectura húngaro Ernő Rubik en 1974.

[5] Hace referencia a la dirección de las manecillas del reloj.

[6] En el sentido semántico de la palabra, la Real Academia Española define "plataforma" como un tablero horizontal, descubierto y elevado sobre el suelo, donde se colocan personas o cosas, en este caso aplica a los elementos de análisis; por su parte, la "dimensión" es cada una de las magnitudes que fijan la posición de un punto en un espacio, para el caso del DUI aplica a los datos. Así, la plataforma es la colocación de los diversos elementos de la metodología en el tablero de $3 \times 3$, mientras que el análisis con datos que dan cuenta de la magnitud del elemento, organizados con una lógica, conforman la dimensión.

[7] Por tratarse de un artículo y con fines de ejemplificación, se resumen los datos más importantes, sin embargo, como parte del Proyecto de Movilidad Inteligente en la Zona Industrial de San Luis Potosí realizado en la Facultad del Hábitat de la Universidad Autónoma de San Luis Potosí existe el detalle de toda la información que incluye más de 30 mil datos que se procesaron para obtener los indicadores que se sintetizan en el cubo. 